\title{
The catabolism of branched-chain amino acids occurs via 2-oxoacid dehydrogenase in Saccharomyces cerevisiae
}

\author{
J. RichaRd DiCKINSON* and IAN W. DAWES \\ School of Biochemistry and Molecular Genetics, University of New South Wales, PO Box 1, Kensington, \\ New South Wales 2033, Australia
}

(Received 11 May 1992; accepted 24 July 1992)

\begin{abstract}
Saccharomyces cerevisiae possesses 2-oxoacid dehydrogenase (EC 1.2.4.4) similar to that found in mammalian cells. The activity is readily detected in cells which have been cultured in a minimal medium containing a branchedchain amino acid. Mutants defective in lipoamide dehydrogenase also lack 2-oxoacid dehydrogenase and are thus unable to catabolize branched-chain amino acids: 2-oxoacids accumulate in the cultures of these cells. The 2-oxoacid dehydrogenase activity is distinct from both 2-oxoglutarate dehydrogenase and pyruvate dehydrogenase, because it could not be detected in assay conditions which permitted the measurement of 2-oxoglutarate dehydrogenase and vice versa. In addition, a strain lacking 2-oxoglutarate dehydrogenase (kgd1::URA3) retained 2-oxoacid dehydrogenase as did a mutant specifically lacking pyruvate dehydrogenase (pda1::Tn5ble). In complex media the specific activity of this enzyme is highest in YEP (yeast extract-peptone)-glycerol and lowest in YEP-acetate and YEP-fructose. 2-Oxoacid dehydrogenase could not be detected in cells which had been transferred to sporulation medium. These results suggest that in $S$. cerevisiae the catabolism of branched-chain amino acids occurs via 2-oxoacid dehydrogenase, not via the 'Ehrlich Pathway'.
\end{abstract}

\section{Introduction}

In eukaryotic cells catabolism of the branched-chain amino acids leucine, isoleucine and valine commences with an aminotransferase reaction to yield $\alpha$-ketoisocaproic acid (4-methyl 2-oxopentanoate), $\alpha$-ketomethylvaleric acid (3-methyl 2-oxopentanoate) and $\alpha$-ketoisovaleric acid (3-methyl 2-oxobutyrate) respectively. These 2-oxoacids then undergo oxidative decarboxylation by branched-chain $\alpha$-ketoacid dehydrogenase [variously called branched-chain oxoacid dehydrogenase or 2-oxoacid dehydrogenase (EC 1.2.4.4)]. This is a multienzyme complex which is structurally similar to the better-known pyruvate dehydrogenase and $\alpha$-ketoglutarate (2-oxoglutarate) dehydrogenase. It consists of three catalytic components: 2-oxoacid dehydrogenase (E1), dihydrolipoyl transacylase (E2) and lipoamide dehydrogenase (E3) (reviewed by Yeaman, 1986).

In mammals pyruvate dehydrogenase, 2-oxoglutarate dehydrogenase and 2-oxoacid dehydrogenase are all

* Author for correspondence. Permanent address: School of Pure and Applied Biology, University of Wales College of Cardiff, PO Box 915, Cardiff CF1 3TL, UK. Tel. (0222) 874000 ext. 5762; fax (0222) 874305 . located in the mitochondrion. Loss of activity of 2-oxoacid dehydrogenase in mammals results in the condition known as Maple Syrup Urine Disease. Not all prokaryotes possess 2-oxoacid dehydrogenase; for instance, Escherichia coli lacks the enzyme and so cannot grow on branched-chain amino acids (Sykes et al., 1987). In Bacillus subtilis the pyruvate dehydrogenase complex also catalyses the decarboxylation of the 2-oxoacids derived from the transamination of leucine, isoleucine and valine (Lowe et al., 1983).

In Saccharomyces cerevisiae 2-oxoacid dehydrogenase has not been described. Decarboxylation of the branched-chain 2-oxoacids is said to proceed via a 'carboxylase' to an aldehyde that is then reduced in a NADH-linked reaction producing the appropriate fusel alcohol (Sentheshanmuganathan, 1960; Woodward \& Cirillo, 1977; Cooper, 1982). The purported pathway in yeast is sometimes referred to as the 'Ehrlich Pathway', because the original ideas were devised by Ehrlich (1904) and slightly modified later (Neubauer \& Fromherz, 1911).

Recently, we have initiated a fresh survey of branched-chain amino acid metabolism in yeast. In addition, we have made an extensive study of the role of lipoamide dehydrogenase ( $L P D 1$ gene product) in this 
organism (Dickinson et al., 1986; Roy \& Dawes, 1987; Ross et al., 1988; Dawes, 1989). Given the vast number of similarities and functional homologies between yeast and many other eukaryotes it seemed unusual that $S$. cerevisiae should differ so greatly in the way that it metabolizes branched-chain amino acids. This prompted the investigation reported here of whether $S$. cerevisiae actually possesses 2-oxoacid dehydrogenase.

\section{Methods}

Strains and cultural conditions. The prototrophic haploid IWD72 $(M A T \alpha)$ from G. Reid (University of Edinburgh, UK) and the transformable haploid IWD3 (MATa ade1-100 his4-519 leu2-3,2-112 ura3-52) were used for most purposes. Strain aW303 $\triangle K G D I$ (MATa ade2-1 his3-11,3-15 leu2-3,2-112 trpl-1 kgdI::URA3) which lacks 2-oxoglutarate dehydrogenase activity (Repetto \& Tzagoloff, 1989) was provided by A. Tzagoloff and B. Repetto (Columbia University, New York, USA). Strain MB204-8C (MATa leu2-3,2-112 trp1-92 ura3-52 pdal::Tn $5 b l e$ ), which lacks pyruvate dehydrogenase activity due to disruption of the gene encoding the El $\alpha$ subunit of that enzyme (Steensma et al., 1990) was provided by T. Wenzel and Y. Steensma (University of Leiden, The Netherlands). Strain IWD5 (MATa

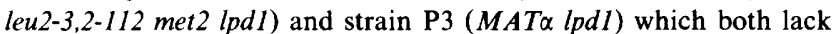
lipoamide dehydrogenase originated from the authors' laboratories (Dickinson et al., 1986). Homothallic diploid S41 (MATa/MATa HO/HO arg4-1/arg4-1 cyhl/cyhl) originated from H. O. Halvorson's laboratory (Brandeis University, Waltham, Mass., USA).

Complex media contained $\left(1^{-1}\right)$ : yeast extract $(10 \mathrm{~g})$, bacteriological peptone $(20 \mathrm{~g})$, adenine $(0 \cdot 1 \mathrm{~g})$ uracil $(0 \cdot 1 \mathrm{~g})$ and the specified carbon source (YEPA, $20 \mathrm{~g}$ potassium acetate; YEPD, $20 \mathrm{~g}$ D-glucose; YEPE, $10 \mathrm{ml}$ ethanol; YEPF, $20 \mathrm{~g}$ D-fructose; YEPG, $30 \mathrm{ml}$ glycerol). Glucose minimal medium comprised $\left(1^{-1}\right)$ : glucose $(20 \mathrm{~g})$, Bacto Yeast Nitrogen Base without amino acids and ammonium sulphate (Difco) $(1.67 \mathrm{~g})$ and ammonium sulphate $(5 \mathrm{~g})$. Branched-chain amino acid minimal medium (BC minimal) contained $\left(1^{-1}\right)$ : Bacto Yeast Nitrogen Base without amino acids and ammonium sulphate $(1.67 \mathrm{~g})$, valine $(20 \mathrm{~g})$ and glucose $(10 \mathrm{~g})$. Minimal media also contained auxotrophic supplements as specifically required by individual strains at $20 \mathrm{mg} \mathrm{l}^{-1}$. All solid media contained agar $\left(20 \mathrm{~g} \mathrm{l}^{-1}\right)$.

For experiments in liquid media cells were grown on a gyrorotatory incubator at $30^{\circ} \mathrm{C}$ in conical flasks filled to $40 \%$ nominal capacity. In studies of sporulation cells raised in YEPD starter cultures were used to inoculate presporulation medium (YEPA $+0 \cdot 1 \%, w / v$, glucose). Sporulation medium contained potassium acetate $(2 \%, w / v)$. Sporulation was induced by the method of Fast (1973).

Preparation of cell extracts and assay of 2-oxoacid dehydrogenase and 2-oxoglutarate dehydrogenase activities. Cells were harvested by centrifugation, resuspended in buffer B ( $50 \mathrm{~mm}$-potassium phosphate pH 7.4, 2 mm-EDTA, 2 mM-2-mercaptoethanol) and disrupted using glass beads as described previously (Dickinson \& Williams, 1986). Aliquots of this homogenate were used immediately as the source of enzyme for the assay of 2-oxoglutarate dehydrogenase activity which was measured in buffer $\mathrm{A}(200 \mathrm{mM}-\mathrm{Tris} / \mathrm{HCl}, \mathrm{pH} 8 \cdot 5$, containing $6 \mathrm{~mm}$ 2-mercaptoethanol) as described by Dickinson et al. (1985). The homogenate was usually also used immediately as the source of 2-oxoacid dehydrogenase, although it could be frozen at $-20^{\circ} \mathrm{C}$ for at least $5 \mathrm{~d}$ without loss of activity. 2-Oxoacid dehydrogenase was assayed in a similar way to 2-oxoglutarate dehydrogenase except that buffer $B$ was used and $\alpha$-ketoisovaleric acid $(14.5 \mu \mathrm{mol})$ was the usual substrate. The limit of sensitivity of the two enzyme assays was 20 pmol substrate consumed $\min ^{-1}$. In one experiment $\alpha$-ketoisocaproic acid and $\alpha$-ketomethylvaleric acid were also used as substrates for 2 -oxoacid dehydrogenase as described below.

Extraction and determination of metabolites by GC. Cells were grown in glucose minimal medium supplemented with either leucine, isoleucine or valine $\left(0.2 \mathrm{~g} \mathrm{l}^{-1}\right)$. When the cells had reached late stationary phase aliquots were harvested in a microcentrifuge. The growth medium was removed to a separate tube to which $\mathrm{H}_{3} \mathrm{PO}_{4}$ was added to a final concentration of $0.1 \mathrm{M}$. After $30 \mathrm{~min}$ at room temperature small quantities $(1-5 \mu \mathrm{l})$ were injected into a Pye Unicam series 106 gas chromatograph. Separation was effected at a temperature of $210^{\circ} \mathrm{C}$ on Chromosorb W. Individual oxoacids were identified by retention times and comparison with authentic compounds.

\section{Results and Discussion}

\section{Mutants lacking lipoamide dehydrogenase accumulate 2-oxoacids}

Growth of strain P3 (which lacks lipoamide dehydrogenase) on glucose minimal medium containing leucine resulted in a high concentration $(1.5 \mathrm{mM})$ of $\alpha$-ketoisocaproic acid in the growth medium. The amount of $\alpha$-ketoisocaproic acid produced indicated virtually stoichiometric conversion of leucine to $\alpha$-ketoisocaproic acid. No $\alpha$-ketoisocaproic acid was detected in cultures of wild-type strains. All strains bearing $l p d l$ mutations grown in this way produce such large amounts of the oxoacid that the cultures have a characteristic goat-like smell. A prototrophic strain was used deliberately for the GC analysis because $\alpha$-ketoisocaproic acid is the penultimate intermediate in the leucine biosynthetic pathway. It was important to demonstrate that the accumulation of $\alpha$-ketoisocaproic acid was due to a $l p d 1$ related defect in a degradative pathway and not merely a partial reversal of the synthetic pathway in the presence of leucine with accumulation of $\alpha$-ketoisocaproic acid due to (e.g.) a leu2 mutation. Subsequent experiments in which lpdI mutants were cultured in the presence of isoleucine or valine indicated the accumulation of $\alpha$-ketomethylvaleric acid and $\alpha$-ketoisovaleric acid (respectively). The fact that branched-chain amino acid degradation was impaired in $l p d l$ mutants suggested that the pathway in yeast involves a 2-oxoacid dehydrogenase complex similar to that in other eukaryotes.

\section{Yeast has a distinct 2-oxoacid dehydrogenase}

Initial experiments involved the growth of prototrophic strain IWD72 to stationary phase in glucose $(1 \%$ w/v) minimal medium supplemented with $2 \%(\mathrm{w} / \mathrm{v})$ leucine. Since at the outset of these investigations nothing was known about the regulation of 2-oxoacid dehydrogenase activity during batch culture, the working hypothesis 
Table 1. Specific activity of 2-oxoacid dehydrogenase and 2-oxoglutarate dehydrogenase assayed in different buffers

Cells of strain IWD72 were cultured for $18 \mathrm{~h}$ in glucose $(1 \%, \mathrm{w} / \mathrm{v})$ minimal medium containing $2 \%(\mathrm{w} / \mathrm{v})$ leucine. The cells were then harvested and 2-oxoglutarate dehydrogenase and 2-oxoacid dehydrogenase were assayed using $\alpha$-ketoglutarate $(\alpha-\mathrm{KGA})$ and $\alpha$-ketoisocaproic acid ( $\alpha$-KIC) as substrates (respectively) in both buffer $A$ and buffer B (see Methods). Approximately $400 \mu \mathrm{g}$ of cell extract was used in these determinations. The results are the means of duplicate determinations $\pm S D$. ND, Not determined.

\begin{tabular}{lcr}
\hline \hline & \multicolumn{2}{c}{$\begin{array}{l}\text { Specific activity [nmol substrate } \\
\text { consumed } \text { min }^{-1}(\mathrm{mg} \text { protein })^{-1} \text { ] }\end{array}$} \\
\cline { 2 - 3 } Substrate & Buffer A & Buffer B \\
\hline $\begin{array}{c}\alpha-\mathrm{KGA} \\
(29 \mu \mathrm{mol})\end{array}$ & $6.64 \pm 0.24$ & $0.00 \pm 0.00$ \\
$\begin{array}{l}\alpha-\mathrm{KIC} \\
(14.5 \mu \mathrm{mol})\end{array}$ & $0.00 \pm 0.00$ & $2.39 \pm 0.08$ \\
$\begin{array}{l}\alpha-\mathrm{KIC} \\
(7.25 \mu \mathrm{mol})\end{array}$ & $0.00 \pm 0.00$ & $1.28 \pm 0.06$ \\
$\alpha-\mathrm{KIC}$ \\
$\begin{array}{l}\left(7.25 \mu \mathrm{mol}+0.1 \mathrm{mM}-\mathrm{Mg}^{2+}\right) \\
\alpha-\mathrm{KIC} \\
\left(7.25 \mu \mathrm{mol}+0.2 \mathrm{mM}-\mathrm{Mg}^{2+}\right)\end{array}$ & ND & $1.05 \pm 0.04$ \\
\hline \hline
\end{tabular}

was that yeast would first utilize the glucose and only start to catabolize leucine once most, or all, of the glucose had been consumed. Hence, it was thought that if yeast possessed 2-oxoacid dehydrogenase, the activity would be most readily apparent after the cells had reached stationary phase in this medium. Yeast had a 2-oxoacid dehydrogenase activity which was biochemically distinct from 2-oxoglutarate dehydrogenase because (branchedchain) 2-oxoacid dehydrogenase activity could not be detected in assay conditions which permitted the measurement of 2-oxoglutarate dehydrogenase and vice versa (Table 1). As assayed in the cell extracts yeast 2-oxoacid dehydrogenase did not require $\mathrm{Mg}^{2+}$; addition of $0.1 \mathrm{mM}$ of this ion produced $18 \%$ inhibition which rose to $44 \%$ inhibition at $0.2 \mathrm{~mm}$. It displayed a pH optimum between 7.2 and 7.8 , which is very similar to the mammalian enzyme.

The 2-oxoacid dehydrogenase activity was genetically distinct from 2-oxoglutarate dehydrogenase because strain aW303 $\triangle K G D 1$, which completely lacks 2-oxoglutarate dehydrogenase due to a disruption in the structural gene of the E1 subunit of this enzyme (Repetto \& Tzagoloff, 1989) has 2-oxoacid dehydrogenase activity, although at lower levels than in the (non-isogenic) wild-type strain IWD72. The 2-oxoacid dehydrogenase activity was also genetically distinct from pyruvate dehydrogenase because strain MB204-8C, which is totally lacking in pyruvate dehydrogenase due to a disruption in the structural gene of the $E 1 \alpha$ subunit of this enzyme (Steensma et al., 1990), has abundant 2-oxoacid dehydrogenase activity. The likely nature of
Table 2. Effect of growth in the presence of different branched-chain amino acids on the activity of 2-oxoacid dehydrogenase towards different 2-oxoacid substrates

Strain IWD72 was cultured for $47 \mathrm{~h}$ in glucose $(1 \%, \mathrm{w} / \mathrm{v})$ minimal medium containing $2 \%(\mathrm{w} / \mathrm{v})$ leucine, isoleucine or valine. The cells were then harvested and 2-oxoacid dehydrogenase was assayed using $\alpha$-ketoisocaproic acid ( $\alpha$-KIC), $\alpha$-ketomethylvalericacid $(\alpha-\mathrm{KMV})$ or $\alpha$-ketoisovaleric acid $(\alpha-\mathrm{KIV})$ as substrate. A maximum of $350 \mu \mathrm{g}$ of cell extract was used in these determinations. The results are the means of duplicate determinations \pm SD.

\begin{tabular}{lccr}
\hline \hline & \multicolumn{3}{c}{$\begin{array}{c}\text { Specific activity }[\mathrm{nmol} \text { substrate } \\
\left.\text { consumed } \text { min }^{-1}(\mathrm{mg} \text { protein })^{-1}\right]\end{array}$} \\
\cline { 2 - 4 } $\begin{array}{c}\text { Amino acid in } \\
\text { growth medium }\end{array}$ & $\alpha-\mathrm{KIC}$ & $\alpha-\mathrm{KMV}$ & $\alpha-\mathrm{KIV}$ \\
\hline Leucine & $2.57 \pm 0.01$ & $<0.01$ & $9.07 \pm 0.04$ \\
Isoleucine & $<0.01$ & $<0.01$ & $3.95 \pm 0.12$ \\
Valine & $2.56 \pm 0.01$ & $1.00 \pm 0.05$ & $11.98 \pm 2.15$ \\
\hline \hline
\end{tabular}

2-oxoacid dehydrogenase in yeast was indicated by the fact that strains bearing $l p d l$ mutations did not possess 2-oxoacid dehydrogenase activity. This confirmed the 2-oxoacid accumulation studies and implies that yeast 2-oxoacid dehydrogenase is a multi-enzyme complex which involves lipoamide dehydrogenase.

To examine whether there are different E1 components for the specific oxidative decarboxylation of the three different 2-oxoacids derived from leucine, isoleucine and valine, separate cultures of cells were grown in each of the three branched-chain amino acids and then 2-oxoacid dehydrogenase activity was determined in each batch of cells using $\alpha$-ketoisocaproic acid, $\alpha$-ketomethylvaleric acid and $\alpha$-ketoisovaleric acid as substrate. The amino acid used in the growth medium for a given batch of cells did not result in a 'preferred' 2-oxoacid substrate for 2-oxoacid dehydrogenase assayed in those cells. In all cases, the highest specific activity resulted with $\alpha$-ketoisovaleric acid as substrate irrespective of the amino acid supplied in the growth medium prior to harvesting and breaking of the cells (Table 2). Accordingly, $\alpha$-ketoisovaleric acid was used as the substrate in all subsequent studies because it allows the greatest sensitivity of detection. Hence the yeast complex appears to be similar to other eukaryotic enzymes and has a single $\mathrm{E} 1$ for the decarboxylation of all branchedchain 2-oxoacids. 2-Oxoacid dehydrogenase could not be detected in isoleucine-grown cells with either $\alpha$-ketomethylvaleric acid or $\alpha$-ketoisocaproic acid as substrate, neither could it be detected in leucine-grown cells using $\alpha$-ketomethylvaleric acid as substrate. Valine-grown cells contained the highest specific activities of 2-oxoacid dehydrogenase and isoleucine-grown cells the least. As with other 2-oxoacid dehydrogenases, $\alpha$-ketomethylvaleric acid served least well as substrate (Table 2). 


\section{Effect of medium composition on the specific activity of 2-oxoacid dehydrogenase}

In batch culture the activity of 2-oxoacid dehydrogenase increased in parallel with cell density (data not shown). In stationary phase cells the highest specific activity [approximately $20 \mathrm{nmol} \alpha$-ketoisovaleric acid consumed $\mathrm{min}^{-1}$ (mg protein $\left.)^{-1}\right]$ was found in cells grown in YEPglycerol. This was rather surprising because one would have expected the specific activity of 2-oxoacid dehydrogenase to have been highest in cells which had been grown in a medium containing a branched-chain amino acid (where the specific activity was only half that found in cells grown in YEP-glycerol). The lowest specific activity was observed in cells which had been grown in YEP-acetate and YEP-fructose: it was only one-half that found in YEP-glucose-grown cells and only about oneeighth the amount in YEP-glycerol-grown cells. Thus it appears that for cells grown in complex media there are similarities in the regulation of 2-oxoacid dehydrogenase and branched-chain amino acid aminotransferase as the relative specific activities vary coordinately in YEPfructose, YEP-glucose, YEP-ethanol and YEP-glycerol (J. R. Dickinson, unpublished). As was also the case with branched-chain amino acid aminotransferase, there is no obvious metabolic advantage to the cell in having a lower specific activity of 2-oxoacid dehydrogenase when grown on YEP-fructose compared to cells grown on YEP-glucose.

The recognition that there is similar regulation of two enzymes (branched-chain amino acid aminotransferase and 2-oxoacid dehydrogenase) which occupy adjacent locations in the same metabolic pathway prompted an investigation of the activity of 2-oxoacid dehydrogenase in diploid strain S41 which had been induced to sporulate. The activity of 2-oxoacid dehydrogenase was below the limit of detection (i.e. less than $20 \mathrm{pmol}$ $\alpha$-ketoisovaleric acid consumed $\min ^{-1}$ ), irrespective of the amount of protein added to the assay. This result is completely opposite to the increase in specific activity of branched-chain amino acid aminotransferase observed when haploid and diploid strains were transferred to sporulation medium (J. R. Dickinson, unpublished). The specific activity of 2-oxoacid dehydrogenase was also low in haploid strain IWD72 which had been grown to stationary phase in YEP-acetate.

It might be argued that the levels of 2-oxoacid dehydrogenase would be expected to be low in media containing the two-carbon compound acetate, because if the pathways of branched-chain amino acid catabolism are the same in yeast as in mammalian systems it would be wasteful biosynthesis for a yeast cell to synthesize an enzyme whose role is to yield (ultimately) intermediates such as acetyl-CoA and succinate. However, this is problematical because branched-chain amino acids will not serve as sole source of nitrogen and carbon in yeast. Furthermore, this explanation does not account for the moderately high level of 2-oxoacid dehydrogenase observed in YEP-ethanol or the low level observed in YEP-fructose. Further research into the details of branched-chain amino acid catabolism is needed before these observations can be rationalized.

It is now clear that $S$. cerevisiae utilizes lipoamide dehydrogenase as an essential component of three multienzyme complexes: pyruvate-, 2-oxoglutarate- and branched-chain 2-oxoacid dehydrogenase. Another multi-enzyme system which contains lipoamide dehydrogenase is the 'glycine cleavage system', which reversibly catalyses the degradation of glycine in animal and plant mitochondria and in prokaryotes (Kikuchi, 1973). Since $S$. cerevisiae has been shown to possess glycine decarboxylase (Ogur et al., 1977), it is tempting to speculate that the $L P D 1$ gene product may also be involved in this.

Much of this research was done whilst J.R.D. visited the University of New South Wales. Financial support from The Royal Society, British Council and United Distillers (which enabled the visit to take place) is gratefully acknowledged. We thank Geoff Kornfeld for stimulating discussions and help at all stages of the work and Nick Balneaves for technical assistance. The late Tony Williams (Cardiff) did the preliminary GC determinations. We are indebted to those colleagues who supplied strains.

\section{References}

COOPER, T. G. (1982). Nitrogen metabolism in Saccharomyces cerevisiae. In The Molecular Biology of the Yeast Saccharomyces cerevisiae. Metabolism and Gene Expression, pp. 211-287. Edited by J. N. Strathern, E. W. Jones \& J. R. Broach. Cold Spring Harbor, NY: Cold Spring Harbor Laboratory.

DAWES, I. W. (1989). Complex regulation of gene expression in Saccharomyces cerevisiae and the implications for biotechnology. Australian Journal of Biotechnology 3, 117-124.

Dickinson, J. R. \& Williams, A. S. (1986). A genetic and biochemical analysis of the role of gluconeogenesis in sporulation of Saccharomyces cerevisiae. Journal of General Microbiology 132, 2605-2610.

Dickinson, J. R., Ambler, R. P. \& DaWes, I. W. (1985). Abnormal amino acid metabolism in mutants of Saccharomyces cerevisiae affected in initiation of sporulation. European Journal of Biochemistry 148, 405-406.

Dickinson, J. R., Roy, D. J. \& Dawes, I. W. (1986). A mutation affecting lipoamide dehydrogenase, pyruvate dehydrogenase and 2-oxoglutarate dehydrogenase activities in Saccharomyces cerevisiae. Molecular and General Genetics 204, 103-107.

EHRLICH, F. (1904). Über das natürliche Isomere des Leucins. Berichte der Deutschen Chemischen Gesellschaft 37, 1809-1840.

FAST, D. (1973). Sporulation synchrony in yeast. Journal of Bacteriology 116, 200-210.

KIKUCHI, G. (1973). Glycine cleavage system. Composition, reaction mechanism, and physiological significance. Molecular and Cellular Biochemistry 1, 169-187.

Lowe, P. N., Hogdson, J. A. \& Perham, R. N. (1983). Dual role of a single multienzyme complex in the oxidative decarboxylation of pyruvate and branched-chain 2-oxo acids in Bacillus subtilis. Biochemical Journal 215, 133-140. 
Neubauer, O. \& Fromherz, K. (1911). Über den Abbau der Aminosäuren bei der Hefegärung. Hoppe-Seyler's Zeitschrift für Physiologische Chemie 70, 326-350.

Ogur, M., liu, T. N., Cheung, I., Paulavicius, I., Wales, W., MehNeRT, D. \& Blaise, D. (1977). 'Active' one-carbon-generation in Saccharomyces cerevisiae. Journal of Bacteriology 129, 926-933.

RePETto, B. \& Tzagoloff, A. (1989). Structure and regulation of $K G D 1$, the structural gene for yeast $\alpha$-ketoglutarate dehydrogenase. Molecular and Cellular Biology 9, 2695-2705.

Ross, J., ReID, G. A. \& DAWES, I. W. (1988). The nucleotide sequence of the LPDI gene encoding lipoamide dehydrogenase in Saccharomyces cerevisiae: comparison between eukaryotic and prokaryotic sequences for related enzymes and identification of potential upstream control sites. Journal of General Microbiology 134, 1131-1139.

RoY, D. J. \& DAwES, I. W. (1987). Cloning and characterization of the gene encoding lipoamide dehydrogenase in Saccharomyces cerevisiae. Journal of General Microbiology 133, 925-933.
Sentheshanmuganathan, S. (1960). The mechanism of the formation of higher alcohols from amino acids by Saccharomyces cerevisiae. Biochemical Journal 74, 568-576.

Steensma, H. Y., Holterman, L., DekKer, I., van Sluis, C. A. \& WENZEL, T. J. (1990). Molecular cloning of the gene for the Ela subunit of the pyruvate dehydrogenase complex from Saccharomyces cerevisiae. European Journal of Biochemistry 191, 769-774.

Sykes, P. J, Burns, G., Menard, J., Hatter, K. \& Sokatch, J. R (1987). Molecular cloning of genes encoding branched-chain keto acid dehydrogenase of Pseudomonas putida. Journal of Bacteriology 169, 1619-1625.

Woodward, J. R. \& Cirillo, V. P. (1977). Amino acid transport in nitrogen-starved cells of Saccharomyces cerevisiae. Journal of Bacteriology 130, 714-723.

Yeaman, S. J. (1986). The mammalian 2-oxoacid dehydrogenases: a complex family. Trends in Biochemical Sciences 11, 293-296. 